\title{
Dynamic analysis and parameter matching simulation system of DTH Hammer
}

\author{
Wang Xueqing, Li Xing, Wang Xiaozhe, Wang Zhongxiang, Gao Chao, Guo \\ Xiangsheng, Wang Yingqi, Shang Yabo, Tian Yuguo
}

School of Mechanics \& Civil Engineering, China University of Mining \& Technology Beijing, Beijing, 100083

wxqease@163.com

Keywords: DTH hammer; computer simulation; dynamic analysis; parameter matching

Abstract: DTH hammer drilling in rock fragmentation has been widely used, but there is still a lack of effective computer-aided design tools. In this study, the dynamic analysis and parameter matching simulation system for down-the-hole hammer was developed. The dynamic process of down-the-hole drilling was elaborated, and the structural parameters of DTH drilling system were analyzed and studied. This work can provide the basis and reference for the economical and efficient design of DTH drilling system.

\section{Introduction}

Some predecessors have carried out a series of related work about the computer simulation study of dynamic process of DTH drilling ${ }^{[1-4]}$. However, the research is mainly focused on the dynamic analysis of the DTH Hammer without yet established a more comprehensive computer simulation $\operatorname{program}^{[5-7]}$, and there is no a software for the evaluation and parameter optimization of the design scheme. So it is difficult to popularize and apply in the design of DTH impact drilling system ${ }^{[8-10]}$. At present, the design of DTH impact drilling system is mainly based on experience and analogy.

In this case, a computer simulation system for dynamic analysis and parameter matching simulation system of DTH Hammer is developed in our study, which can be used as a computer-aided design method.

\section{Principle of algorithm}

DTH impactor piston movement equation:

$$
M_{P} \frac{d^{2} x}{d t^{2}}=F(t)=P_{1} A_{1}-P_{2} A_{2}-F_{Q}
$$

Where: $M_{P}-$ Piston quality; $x$-Piston displacement; $t$-Time; $P_{1} 、 P_{2}-$ Front and rear chamber pressure; $A_{1} 、 A_{2}-$ Front and rear chamber pressure area; $F_{\mathrm{Q}}-$ Tail force.

To divide the front and back cavity gas distribution process into 5 intervals (Fig. 1 input interface), the change of gas in the movement of the piston is a variable process, the equations of motion are second-order nonlinear differential equations, which can be solved by numerical methods, the quasi-stable state technique is used to determine the unit displacement S., divide the piston motion into sufficiently short micro segments. In each micro segment, the gas state is constant then the force of the piston is a constant force, the motion of the piston can be regarded as acceleration, the values of the pressure in each zone are shown in Table 1. 
Table 1: Each Section Pressure Value

\begin{tabular}{|c|c|c|c|c|c|}
\hline Intervals & I & II & III & IV & V \\
\hline$P_{1}(N)$ & $P_{I}$ & $P_{I}$ & $P_{1}(N)=P_{1}(N-1)\left[\frac{L_{D}+x(N-1)}{L_{D}+x(N-1)+S}\right]^{1.3}$ & $P_{0}$ & $P_{I}$ \\
\hline$P_{2}(N)$ & $P_{0}$ & \multicolumn{2}{c|}{$P_{2}(N)=P_{2}(N-1)\left[\frac{L U L-x(N-1)}{L U L-x(N-1)-S}\right]^{1.3}$} & $P_{I}$ \\
\hline
\end{tabular}

$\mathrm{P}_{\mathrm{I}}$ and $\mathrm{P}_{0}$ in the table are inlet and outlet pressures, according to the equation (1), the numerical solution is performed, and the calculation is performed step by step. Each cycle is obtained until the movement of the piston tends to a stable-state motion (approaching the limit cycle), and the calculation result and the stable-state motion curve are given (the output interface in Fig. 3).

\section{Input Interface}

The effect of the DTH hammer Can be study by changing the parameters.

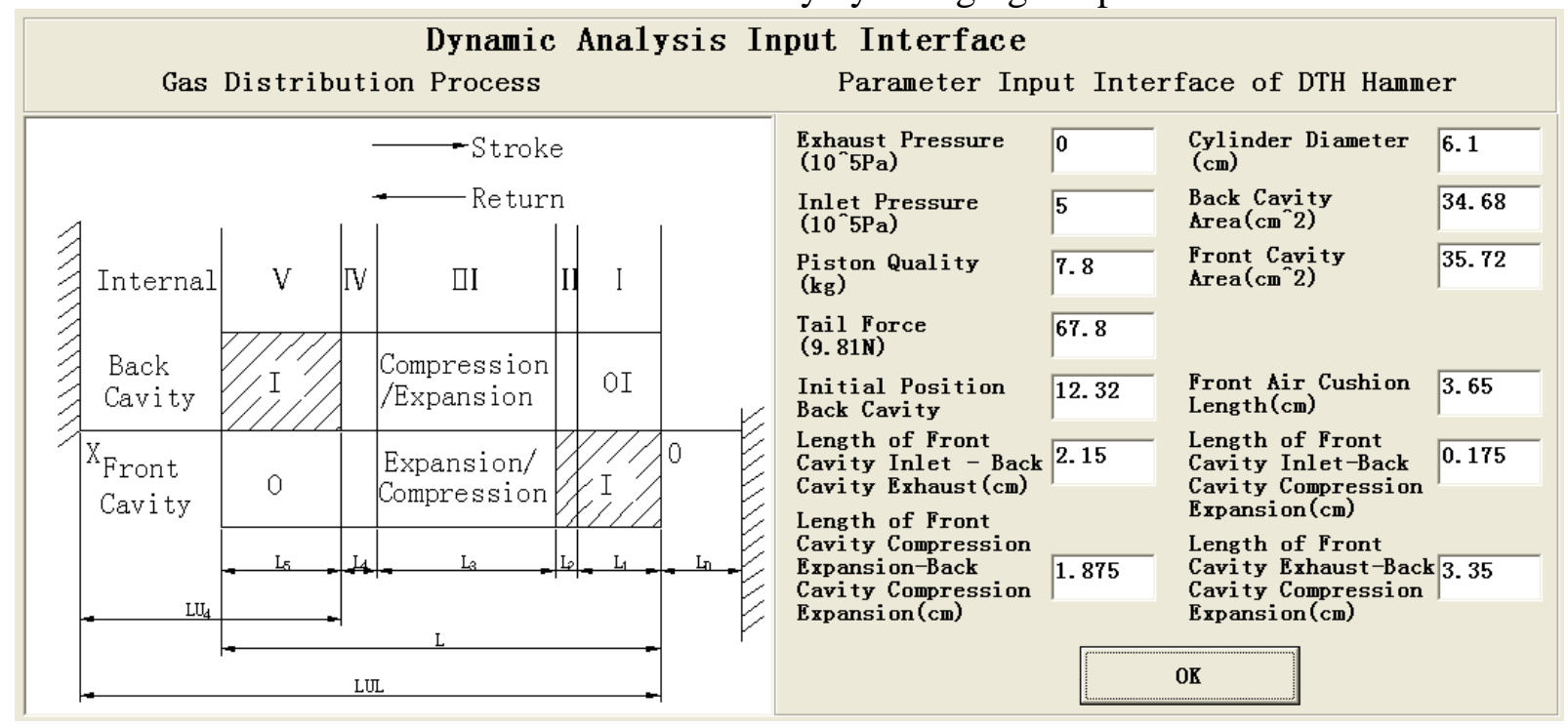

Fig. 1 Dynamic Analysis Input Interface

Fig. 1: $L_{1} —$ Length of Front cavity Inlet- Back Cavity Exhaust, $\mathrm{cm} ; L_{2}$ — Length of Front cavity Inlet- Back Cavity Compression Expansion, $\mathrm{cm} ; L_{3} \_$Length of Front cavity Compression Expansion-Back Cavity Compression Expansion, $\mathrm{cm} ; L_{4}$ _L Length of Front cavity Exhaust- Back Cavity Compression Expansion, $\mathrm{cm} ; L_{5}$ _Length of Front cavity Exhaust- Back Cavity Inlet, cm; $L_{\mathrm{D}}$ _ Front Air Cushion Length, $\mathrm{cm} ; \mathrm{LU}_{4} \_$Length of Back cavity at the end of phase IV, cm; $L U L-$ Length of Back cavity in the Beginning, cm; $L-$ Piston throw, $L=L_{1}+L_{2}+L_{3}+L_{4}+L_{5}$.

\section{Output Interface}

Fig. 2 (a) - (f) shows the gradual transition of the piston from the initial position to a stable state of motion. Fig. $2(\mathrm{~g})$ is the stable motion state of the piston, i.e., the reciprocating cycle of the piston in the limit circle of the outer ring. Fig. 2 (h) shows the displacement and time motion curves of piston in stable state motion. Fig. 2 (I) shows the calculation results of the relevant parameters of the piston in stable state motion.

Fig. 3 shows the full output interface of the dynamic analysis and parameter matching computer simulation system of DTH hammer. 


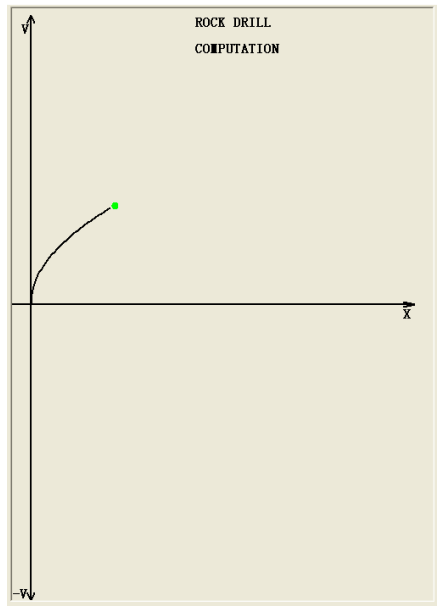

(a) Initial stage

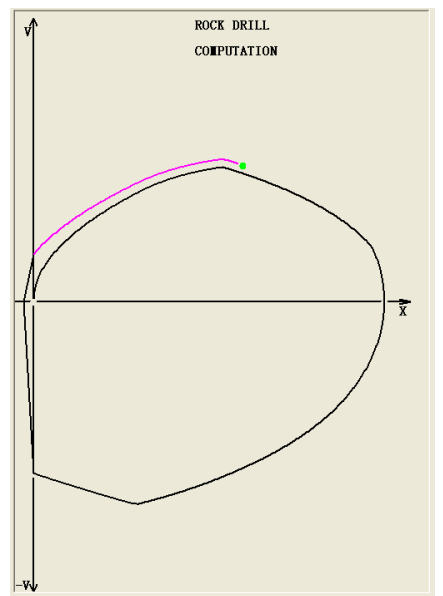

(d) Start the second track

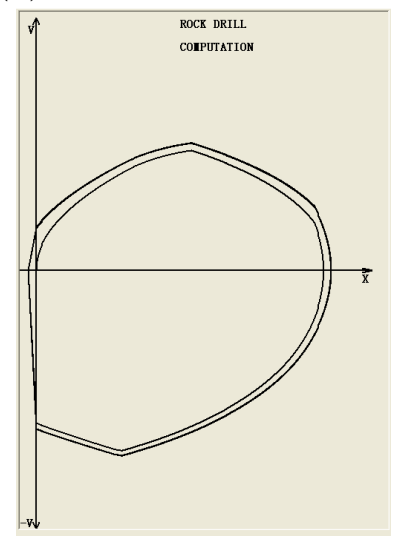

(g) Loop around the outside

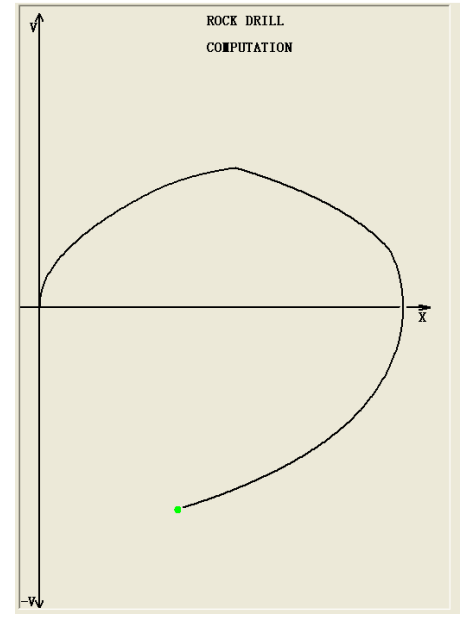

(b) Initial trajectory phase
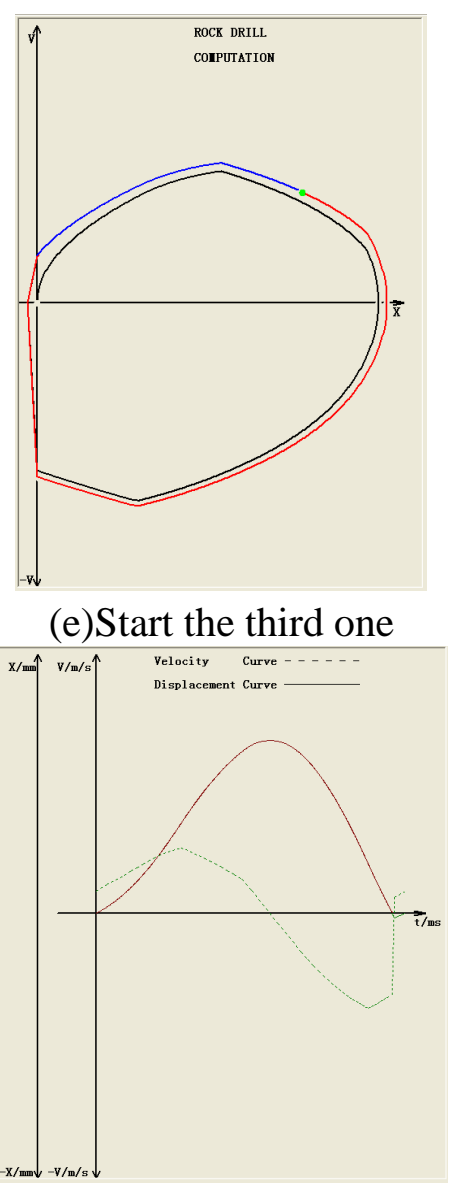

(h) Stable state motion curve

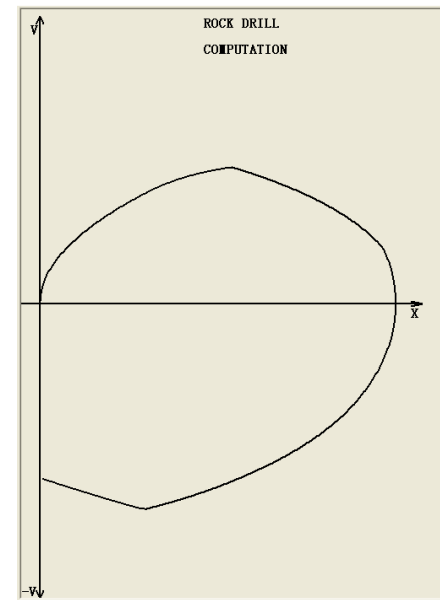

(c) Initial trajectory completion phase

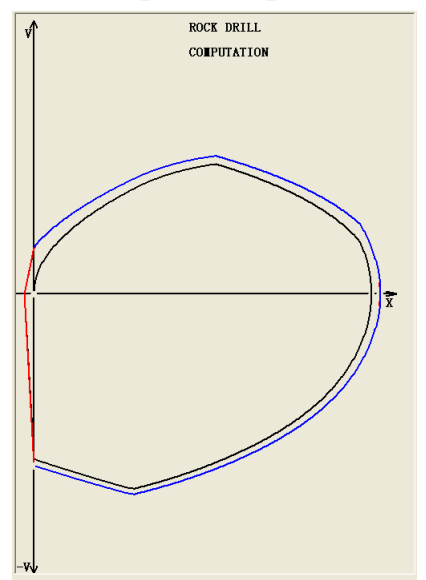

(f) Complete the third track

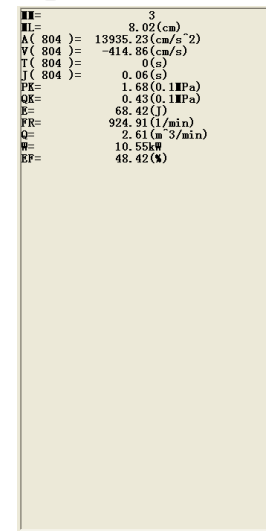

(i) Calculate results

Fig. 2 Dynamic Analysis and Parameter Matching Output Interface Processes 


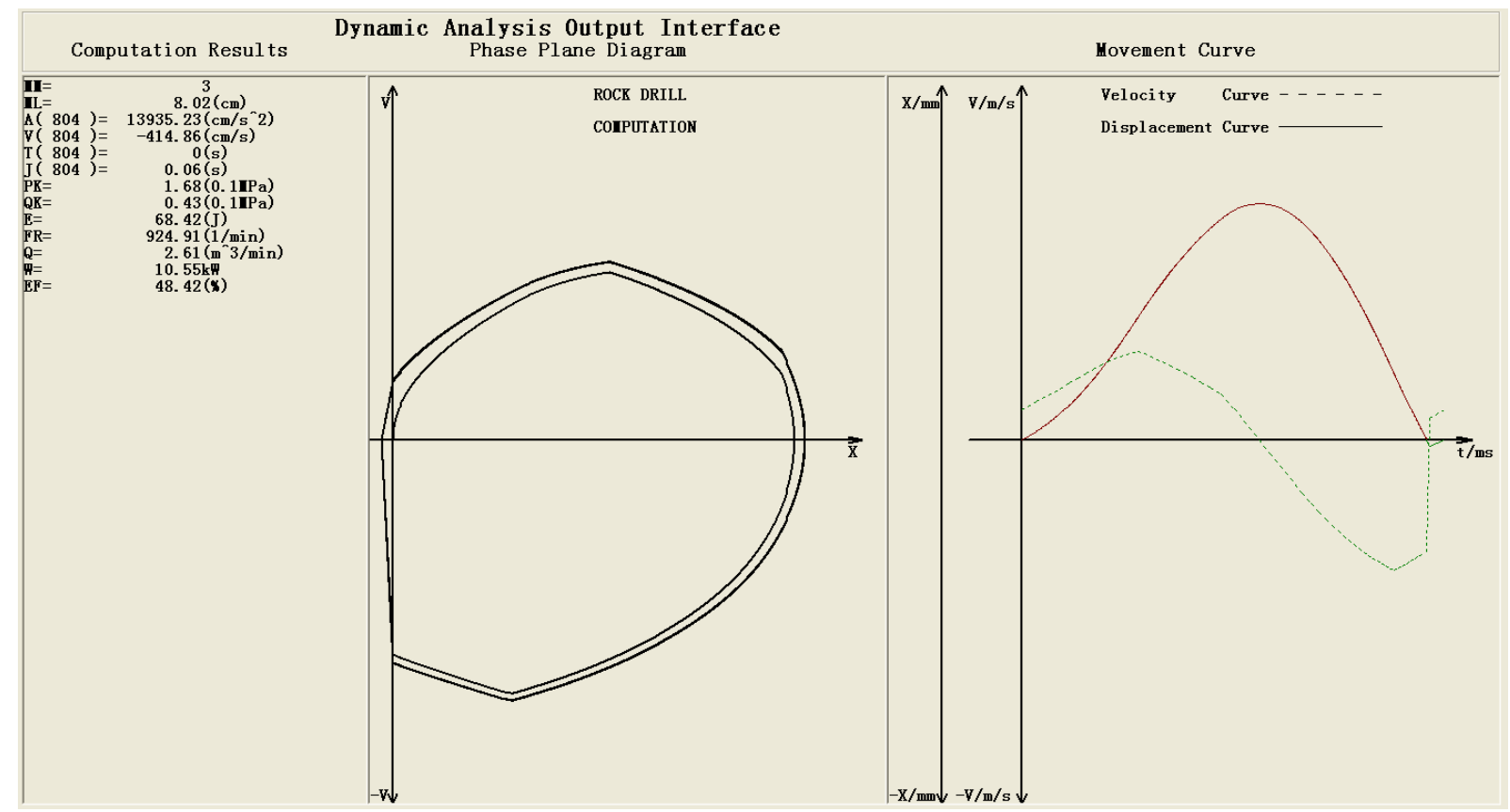

Fig. 3 Dynamic Analysis and Parameter Matching Output Interface

\section{Conclusion}

The simulation system uses the computer simulation method to comprehensively analyze the dynamics and parameter matching process of the downhole drilling system, which can give the influence of various parameters on the performance of the downhole drilling system. It can be used for the optimization design and efficiency evaluation of the product.

\section{References}

[1] Wang J. Study and Application on Drilling Technique of Deep Hole Fluid-powered DTH Hammers. Site Investigation Science \& Technology, 2011.

[2] Changgen $\mathrm{Bu}$, Yegao Qu, Zhiqiang Cheng, et al. Numerical Simulation of Impact on Pneumatic DTH Hammer Percussive Drilling. Journal of Earth Science, 2009, 20(5):868-878.

[3] Jian Z. The Research on Hydro Efflux DTH Hammer Applying to Oil and Gas Exploration Drilling. World Geology, 1998.

[4] Yin K, Jiang R, Lai Z. Drilling Technology of Air driven DTH Hammer. World Geology, 1999.

[5] Fan L M, Yin K, Zhang Y G, et al. Numerical investigation of geometry parameters on side-ejector DTH hammer RC bit. Journal of Central South University, 2011, 42(1):220-226.

[6] Yin K, Jiang R. Reverse Circulation DTH Hammer Drilling Technique and Its Application. Exploration Engineering, 1996, 14:259-264.

[7] Yu-Bei L U. Application of Air DTH Hammer Technology to Fast Drilling in Red Beds of Yunnan Province. Geology \& Exploration, 2011, 47(2):309-315.

[8] Yin Q, Yin K, Liu H, et al. Application test of DTH hammer reverse circulation drilling technique in a hydropower station. Jilin Daxue Xuebao, 2014, 44(3):961-968.

[9] Suo Z W, Yin K, Ke-Li X U. Mathematical Model and Synthetic Analysis of Hydro-Efflux DTH Hammer inside Power Process. Journal of Jilin University, 2007, 37(01):200-203.

[10] Liu J, Yin K. Development of SGQ-320 Type Reverse Circulation DTH Hammer Used in Gas Drilling. Petroleum Drilling Techniques, 2012, 40(1):114-118. 